\title{
Research on Modelling and Stability Characteristics of Electric Traffic Energy System Based on ZVS-DAB Converter
}

\author{
Ayiguzhali Tuluhong $\mathbb{D}^{1,2}$ Weiqing Wang $\mathbb{D}^{1,3}$ Yongdong $\mathrm{Li}^{1,}{ }^{2}$ Haiyun Wang, ${ }^{1,3}$ and Lie Xu${ }^{2}$ \\ ${ }^{1}$ Electrical Engineering, Xinjiang University, Urumqi 830046, Xinjiang, China \\ ${ }^{2}$ Electrical Engineering, Tsinghua University, Beijing 100084, China \\ ${ }^{3}$ Research Center of Renewable Energy Power Generation and Grid Control Engineering, Ministry of Education, \\ Xinjiang University, Urumqi 830046, China \\ Correspondence should be addressed to Weiqing Wang; 1374457465@qq.com
}

Received 10 February 2020; Revised 13 April 2020; Accepted 14 May 2020; Published 28 May 2020

Academic Editor: Luca Maresca

Copyright (C) 2020 Ayiguzhali Tuluhong et al. This is an open access article distributed under the Creative Commons Attribution License, which permits unrestricted use, distribution, and reproduction in any medium, provided the original work is properly cited.

\begin{abstract}
We study and describe mostly used traditional simplified circuits for full-bridge Zero Voltage Switching-Dual Active Bridge (ZVS$\mathrm{DAB})$ converter and deduce their mathematical model. On this basis, we propose a high-frequency (HF) mathematical model, which takes into account conduction loss and HF characteristics of the ZVS-DAB converter model. We compare the static and dynamic stabilities of the traditional and the proposed HF mathematical model by simulation. Finally, the high-frequency planar transformer (HFPT) with good heat dissipation and the wide band gap (WBG) semiconductor SiC switches with fast switching speed are employed to build a $4.4 \mathrm{kw}, 40 \mathrm{KHz}$ experimental prototype to verify the effectiveness of the improved HF circuit of ZVS-DAB converter. The results show that the proposed HF mathematical model is superior to the traditional one, and it fully considers the HF characteristics of the circuit and effectively improves the HF oscillation, DC bias, and waveform distortion of the ZVS-DAB converter.
\end{abstract}

\section{Introduction}

Energy crisis and environmental pollution are two major social problems facing the world today, and vehicle exhaust, as traffic pollution, is one of the main culprits of environmental pollution [1]. With the development of electric transportation technology, traditional fuel transportation as an important way of nonrenewable energy consumption is gradually replaced by new energy transportation. Electric vehicles, multielectric aircraft, maglev high-speed trains, and vacuum high-speed trains, as representatives of new energy vehicles, do not rely on fossil fuels, but also have little environmental pollution. In the field of electrified transportation, more and more power electronic equipment and power supply system are being pursued to be miniaturized in size and light in weight. This trend has made new energy transportation power supply system, power electronic transformer (PET), which has become an important development direction of power electronic industry [2-4]. As one of the important parts of new energy transportation and the hub of the smart grid and the energy Internet, PET can improve the power density and reduce the volume and weight of power supply after high frequency (HF).

Compared with the same power level Si-based MOSFET, the wide band gap (WBG) semiconductor device, SiC-based MOSFET, has the advantages of fast switching speed and small on resistance, which will reduce the core volume, improve the working frequency of the transformer, reduce the system weight and volume, improve the energy density and system efficiency, and accelerate the HF development of PET. At present, the price of WBG semiconductor switches is decreasing every year, and it has begun to replace the market of Si-based switches. It is an inevitable trend to replace Si-based switches with WBG switches, such as $\mathrm{SiC}$ $[5,6]$. In order to improve the efficiency of PET, the isolated DC-DC converter with soft switches is generally used. The 
isolated DC-DC converter can be divided into nonresonant dual active bridge (DAB) and resonant mode (LLC) converters. The performance comparison between $\mathrm{DAB}$ and LLC converters is shown in Table 1. In 1991, German scholar Rik W. Doncker put forward the concept of DAB, which can realize the controlled power transmission on both sides of the isolation transformer by phase-shifting control and then become one of the key core technologies of power electronic transformer [7].

By the end of the twentieth century, Chinese researchers developed a $2 \mathrm{kw}$ power supply independently, and it was used in the field of communication with the phase-shifting full bridge soft switching technology, the module efficiency was $93 \%$, and the weight of the product with PWM technology was fully reduced by $40 \%$. Because the phase-shifting full bridge ZVS-DAB converter can complete the conversion of large and medium power at HF and has high efficiency, simple structure [8], and good dynamic characteristics, it has attracted great attention of scholars in the power supply field at home and abroad in recent years [9-12].

In this paper, a new $\mathrm{SiC}$ device is used, and the proportion of PET's turn-off loss in the total loss of the system is very low, so it is more economical to choose $\mathrm{DAB}$ converter. In addition, the utility of high frequency planar transformer (HFPT) in ZVS-DAB has also accelerated the HF development and volume miniaturization of the PET $[13,14]$. However, there are some disadvantages in the application of WBG and HFPT in ZVS-DAB, such as HF oscillation, HF loss, and DC bias, which make the system unstable, and it needs to be solved. The oscillation is caused by the parasitic parameters of ZVS-DAB, such as parasitic inductance and capacitance of semiconductor devices, HFPT, as well as PCB at HF [15]. In view of this, in reference [15], scholars have analyzed that the HF parasitic capacitance of HFPT causes serious distortion of the light load current and voltage waveforms of the LLC converter and brings regulation issues for the converter. In essence, the parasitic capacitance of HFPT can be reduced, and the output waveform can be reached stable by improving the transformer structure. In [16], researchers eliminate the HF oscillation in ZVS-DAB via optimizing $\mathrm{dv} / \mathrm{dt}$, so as to achieve system stability. In addition to these problems, HF also causes DC bias. In [17], the deviation between the transient current and the steadystate current caused by the DC bias is discussed, and the transient phase-shifting strategy is adopted to eliminate the transient DC bias.

Generally, in order to simplify the calculation, the theoretical and simulation analysis of the ZVS-DAB converter is generally carried out. Although the simulation results are consistent with the experimental results, some errors still occur. In this paper, in order to avoid errors and fully reflect the HF characteristics of the ZVS-DAB converter, we study nonmechanism models of the ZVS-DAB converter and propose a HF mathematical model for the ZVS-DAB converter, which fully considers turn-on loss and HF characteristics of the ZVS-DAB converter such as oscillation, parasitic capacitance, and inductance in the electrified transportation energy system. This structure is organized as follows: Section 2 introduces three simplified circuit topological models of the ZVS-DAB converter briefly. In Section 3, their mathematical models are deduced, respectively. Then, on this basis, section IV analyzes and compares the static and dynamic stability characteristics of the traditional ZVS-DAB converter model and the proposed HF-ZVS-DAB converter model by simulation and experimental prototype in detail. Finally, Section VII concludes the paper.

\section{ZVS-DAB Converter Topology Equivalent Circuit}

2.1. ZVS-DAB Converter. The main circuit topology of the electric traffic energy system based on the traditional ZVS$\mathrm{DAB}$ converter is shown in Figure 1, where $L 1$ and $L 2$ represent the primary and secondary phase-shifting inductors (PSI), and $V 1, V 2$ correspond to the input and output $\mathrm{DC}$ voltages of the $\mathrm{DAB}$, and $U_{\mathrm{p}}$ and $U_{\mathrm{s}}$ are the primary and secondary AC voltages of the HFPT. The switching frequency is $40 \mathrm{kHz}$, and the transformation ratio $k$ is set to 3. $D_{1}, D_{2}, \ldots, D_{8}$, and $C_{1}, C_{2}, \ldots, C_{8}$ are body diodes and resonant capacitors connected in parallel at both ends of $8 \mathrm{SiC}$ MOSFETs $\left(Q_{1}, Q_{2}, \ldots, Q_{8}\right)$, respectively. After expanding the HFPT topology, the complete equivalent mechanism model can be obtained, where $R_{p}$ and $L_{p}$, and $R_{s}$ and $L_{s}$ represent the resistance and leakage inductance of the primary and secondary windings of the HFPT respectively; $R_{m}$ is the equivalent core loss resistance; $L_{m}$ is for the equivalent core magnetizing inductance generated in the primary side; $C_{1}$ and $C_{2}$ refer to the primary and secondary intrawinding capacitance, and $C_{12}$ is the primary and secondary interwinding capacitance; $I_{p}$ and $I_{s}$ are the primary and secondary currents of the HFPT.

For ZVS-DAB converters, the switches are alternately connected in pairs and the upper and lower switches of the same bridge work in the mutual intermittent state. For a given power, the current of full bridge $\mathrm{DAB}$ converter is only $1 / 2$ of that of half bridge $\mathrm{DAB}$ converter, and the output power of the full bridge $D A B$ converter is twice that of the half bridge $\mathrm{DAB}$ converter under the same switch current and the same switch voltage. Therefore, full bridge $D A B$ converter is usually used in the occasion of a high power supply. In addition, the full bridge $\mathrm{DAB}$ converter only needs one smoothing capacitor, while the half bridge converter needs two smoothing capacitors.

2.2. Equivalent Circuit of the ZVS-DAB Converter. For the convenience of calculation, the model of ZVS-DAB converter, as shown in Figure 1, is usually simplified into the circuit shown in Figure 2, where two controllable voltage sources $V 1$ and $V 2$ are connected with an inductor $L$. the voltage difference between the inductor is adjusted by controlling phase-shifting angle and duty cycle of the two voltage sources, so as to control the inductor current $i_{L}$, and then control the current and power of the ZVS-DAB converter.

In an ideal case, all losses are ignored in the traditional ZVS-DAB converter, and the DC-link capacitors and two 
TABLE 1: Performance comparison between DAB converter and LLC resonant converter.

\begin{tabular}{lcc}
\hline Parameter & DAB & LLC \\
\hline External capacitor & No (small volume) & Yes (large volume) \\
Voltage controllability & High & Low \\
Total ZVS & Yes & Yes \\
Secondary side ZCS & No & Yes \\
Parallel current-sharing & Easy & Difficult \\
Antimagnetic saturation & Software & Hardware \\
Current stress & Lower & Higher \\
Suppression of DC second harmonic & Yes & No \\
Control points & PI voltage closed loop, antimagnetic saturation & Power switching, load sharing \\
\hline
\end{tabular}

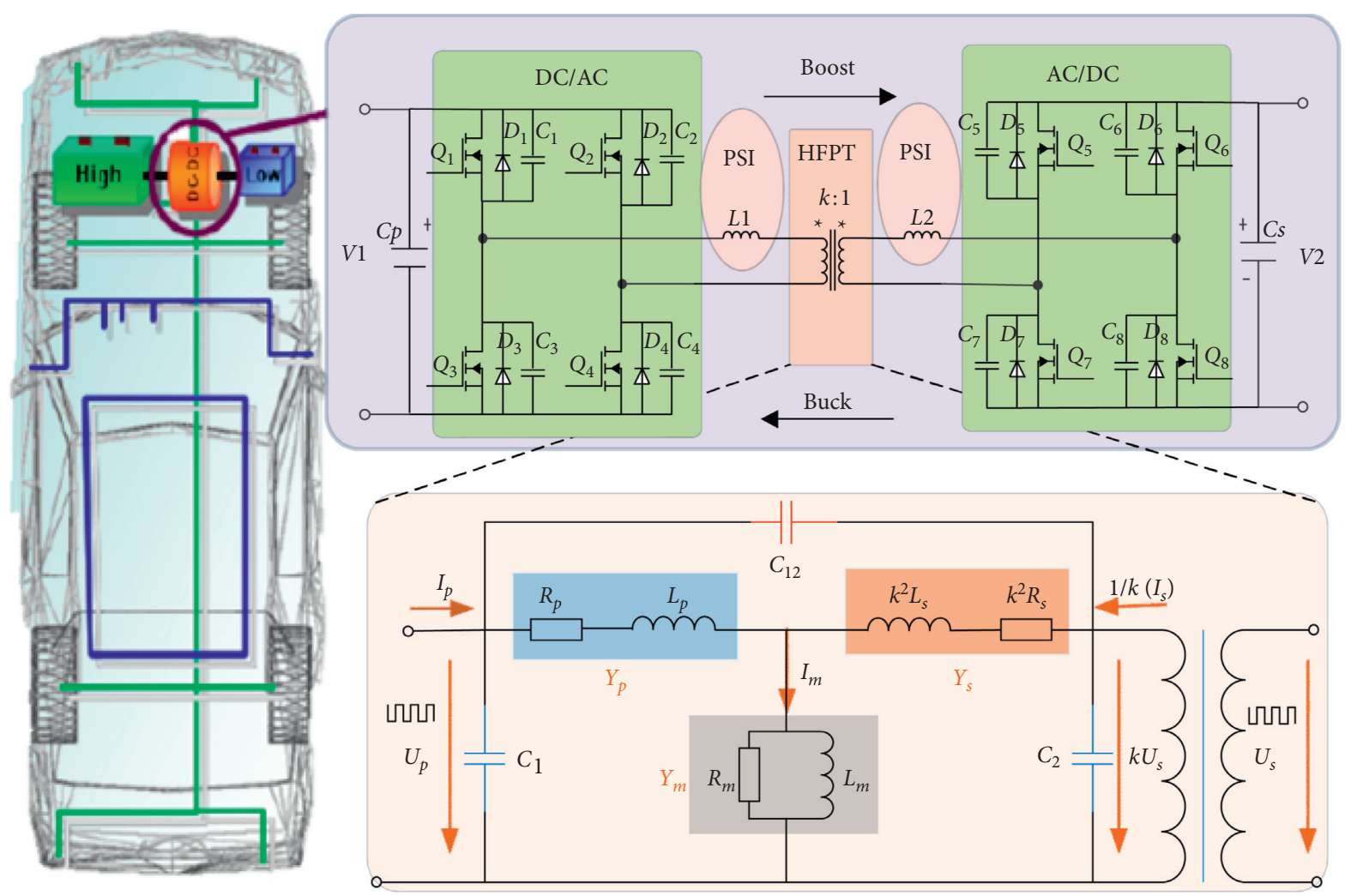

Figure 1: Topology circuit of the electric traffic energy system based on the ZVS-DAB converter.

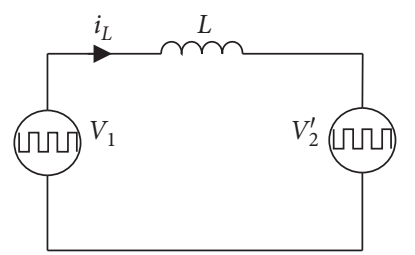

FIGURE 2: : Simplified circuit of the traditional ZVS-DAB converter.

full bridges on both sides can be equivalent to two three-level voltage sources, and the HFPT equivalent to a leakage inductance $[18,19]$, which acts as a power transfer component. The most commonly employed modulation algorithm of ZVS-DAB converter is SPS control, so the equivalent threelevel voltage sources degrade to two-level square wave voltage sources with duty cycle of $50 \%$.
However, the traditional equivalent circuit cannot fully reflect $\mathrm{HF}$ characteristics of the ZVS-DAB converter since it ignores many factors, that is, $\mathrm{HF}$ loss, HF oscillation, $\mathrm{HF}$ transmission, and so on. In view of this, scholars have studied and proposed different simplified circuits. Figure 3 is a simplified ZVS-DAB circuit considering the conduction loss of converter researched by ETH scholar Florian Krismer. The HF loss model of ZVS-DAB can be established through mathematical model, but the HF oscillation characteristics of ZVS-DAB cannot be reflected accurately. Based on the circuit in Figure 3, this paper studies the simplified circuit of the ZVS-DAB converter, which is shown in Figure 4, composed of lumped inductance, equivalent capacitance, and lumped resistance, and deduces the mathematical model of inductance current, that is demonstrated in formula (10). This proposed mathematical model fully 


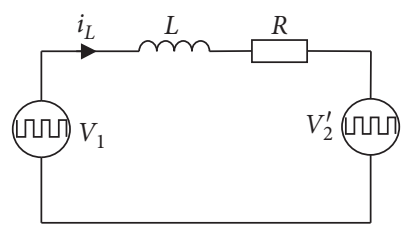

Figure 3: Improved simplified circuit of the ZVS-DAB converter, which considers conduction loss.

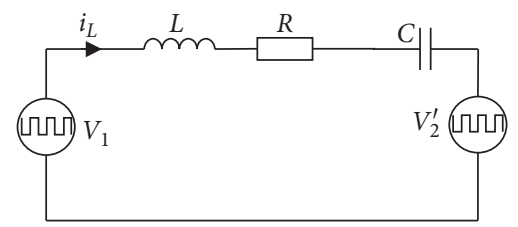

FIGURE 4: Improved HF simplified circuit of the ZVS-DAB converter.

embodies the HF characteristics of the ZVS-DAB converter, and the results are closer to the real model.

\section{Mathematical Model of the ZVS- DAB Converter}

There are some state of the art modulation schemes for ZVSDAB converters [20]. However, SPS control is a commonly used phase-shifting control strategy for ZVS-DAB converters because of its simplicity. And its two H-bridge inner phase-shifting values are 0.5 , so power transmission is controlled only via changing the outer phase shift. Under steady-state conditions, the primary and secondary side voltage and current waveforms are shown in Figure 5. It can be seen that the voltage and current values will repeat at each half cycle, as shown in the following formula:

$$
\left\{\begin{array}{l}
V_{1}\left(t+\frac{T_{S}}{2}\right)=-V_{1}(t) \\
V_{2}^{\prime}\left(t+\frac{T_{S}}{2}\right)=-V_{2}^{\prime}(t) \\
i_{L}\left(t+\frac{T_{S}}{2}\right)=-i_{L}(t)
\end{array}\right.
$$

The mathematical models of Figures 2-4 can be derived, respectively, according to Figure 5 and the above formula. In all expressions, $t_{1}=\varphi T_{s} / 2$ and $V_{1}, V_{2}^{\prime}$ are two square waves with a duty cycle of $50 \%$, and phase-shifting angle $\varphi(0,1), V_{2}^{\prime}$ is the voltage converted from the secondary side to the primary side, $L$ is the sum of the primary and secondary phase-shift inductance and leakage inductance.
3.1. Mathematical Model of the Traditional Simplified ZVS$D A B$ Converter. The following formula can be deduced from Figure 2:

$$
i_{L}(t)=i_{L}\left(t_{0}\right)+\frac{1}{L} \int_{t_{0}}^{t}\left(V_{1}-V_{2}^{\prime}\right) \mathrm{d} t, \quad t_{0}<t .
$$

Then, the steady-state current of traditional ZVS-DAB converter within one switching cycle Ts is as follows:

$$
i_{L}(t)=\left\{\begin{array}{l}
\frac{(1-2 \varphi) V_{2}^{\prime}-V_{1}}{4 L} T_{s}+\frac{V_{1}+V_{2}^{\prime}}{L} t, \quad t \in\left[0, t_{1}\right], \\
\frac{V_{2}^{\prime}-(1-2 \varphi) V_{1}}{4 L} T_{s}+\frac{V_{1}-V_{2}^{\prime}}{L}\left(t-t_{1}\right), \quad t \in\left[t_{1}, \frac{T_{s}}{2}\right], \\
-i_{1}\left(t-\frac{T_{s}}{2}\right), \quad t \in\left[\frac{T_{s}}{2}, T_{s}\right] .
\end{array}\right.
$$

According to equation (1), the power transmission characteristics of ZVS-DAB converter under SPS control mode are as follows:

$$
P=\frac{1}{T_{s}} \int_{0}^{T_{s}} u_{1} i_{1} \mathrm{~d} t=\frac{V_{1} V_{2}^{\prime} T_{s}}{2 L} \varphi(1-\varphi) .
$$

3.2. Mathematical Model of the Improved ZVS-DAB Converter considering Conduction Loss. The following formula can be deduced according to Figure 3:

$$
i_{L}(t)=i_{L}\left(t_{0}\right)+\frac{1}{L} \int_{t_{0}}^{t}\left[V_{1}-V_{2}^{\prime}-i_{L}(t) R\right] \mathrm{d} t, \quad t_{0}<t .
$$




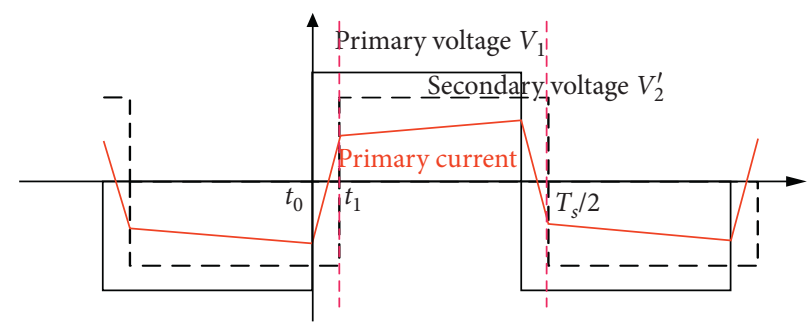

FIgURE 5: Output waveform of the ZVS-DAB converter under SPS control.

The steady-state current of ZVS-DAB converter considering conduction loss in one switching cycle Ts is as follows:

$$
i_{L}(t)=\left\{\begin{array}{l}
e^{-t / \tau} i_{L, 0}+\left(1-e^{-t / \tau}\right) \frac{V_{1}+V_{2}^{\prime}}{R}, \quad t \in\left[0, t_{1}\right], \\
e^{-\left(t-t_{1}\right) / \tau} i_{L}\left(t_{1}\right)+\left(1-e^{-\left(t-t_{1}\right) / \tau}\right) \frac{V_{1}-V_{2}^{\prime}}{R}, \quad t \in\left[t_{1}, \frac{T_{s}}{2}\right], \\
-i_{1}\left(t-\frac{T_{s}}{2}\right), \quad t \in\left[\frac{T_{s}}{2}, T_{s}\right],
\end{array}\right.
$$

where $\tau=L / R$,

$i_{L}(t)=i_{L}\left(t_{0}\right)+\frac{1}{L} \int_{t_{0}}^{t}\left[V_{1}-V_{2}^{\prime}-i_{L}(t) R-u_{c}(t)\right] \mathrm{d} t, \quad t_{0}<t$.

$\frac{i_{L, 0}=V_{1}+V_{2}^{\prime}-V_{1} e^{T_{s} / 2 \tau}+V_{2}^{\prime} e^{T_{s} / 2 \tau}-2 V_{2}^{\prime} e^{\varphi T_{s} / 2 \tau}}{R\left(e^{\left(T_{s} / 2 \tau\right)+1}\right)}$.

The steady-state current of ZVS-DAB converter considering HF loss and oscillation in one switching cycle Ts is

3.3. Mathematical Model of the Improved HF ZVS-DAB as follows:

Converter. From Figure 4, we can get the following formula:

$$
i_{L}(t)=\left\{\begin{array}{l}
\left(\lambda_{1} e^{\lambda_{1} t}+\lambda_{2} e^{\lambda_{2} t}\right)\left(i_{L, 0}+\left(V_{1}+V_{2}^{\prime}\right) C\right), \quad t \in\left[0, t_{1}\right], \\
\left(\lambda_{1} e^{\lambda_{1}\left(t-t_{1}\right)}+\lambda_{2} e^{\lambda_{2}\left(t-t_{1}\right)}\right)\left(i_{L}\left(t_{1}\right)+\left(V_{1}-V_{2}^{\prime}\right) C\right), \quad t \in\left[t_{1}, \frac{T_{s}}{2}\right], \\
-i_{1}\left(t-\frac{T_{s}}{2}\right), \quad t \in\left[\frac{T_{s}}{2}, T_{s}\right],
\end{array}\right.
$$

where $\quad \lambda_{1,2}=-(R / 2 L) \pm \sqrt{\left(R^{2} / 4 L^{2}\right)-(1 / C L)}, \quad \sigma_{1}=$ $\lambda_{1} \mathrm{e}^{-\left(\lambda_{1} \mathrm{~T}_{s}(\varphi-1) / 2\right)}+\lambda_{2} \mathrm{e}^{-\left(\lambda_{2} \mathrm{~T}_{s}(\varphi-1) / 2\right)}$ and

$$
i_{L, 0}=-\frac{\left(C\left(V_{1}-V_{2}^{\prime}\right)+C\left(\lambda_{1} e^{\lambda_{1} \varphi T_{s} / 2}+\lambda_{2} e^{\lambda_{2} \varphi T_{s} / 2}\right)\left(V_{1}+V_{2}^{\prime}\right)\right) \sigma_{1}+C\left(V_{1}+V_{2}^{\prime}\right)\left(\lambda_{1}+\lambda_{2}\right)}{\lambda_{1}+\lambda_{2}+\left(\lambda_{1} e^{\lambda_{1} \varphi T_{s} / 2}+\lambda_{2} e^{\lambda_{2} \varphi T_{s} / 2}\right) \sigma_{1}} .
$$




\section{Simulation and Experimental Verification of the ZVS-DAB Converter}

The model parameters used in the simulation and experimental analysis of the ZVS-DAB model are tabulated in Table 2.

4.1. Simulation Results of the ZVS-DAB Converter. In this paper, we compare the stability of the HF mathematical model and the traditional mathematical model of the ZVSDAB converter through simulation and experiment. On the experimental setup, the WBG semiconductors generate parasitic inductance at HF, which leads to voltage spikes, and the HFPT generates parasitic capacitance at HF, which leads to current spikes. Moreover, the interaction between these parasitic inductance and capacitance distorts the voltage and current waveforms and leads to regulation issues and oscillations for the ZVS-DAB converter [15]. However, there are no signs of these phenomena during the simulation, so the static stability of the ZVS-DAB converter is analyzed in the simulation, and the dynamic stability is studied in the experiment. Figure 6 is the voltage and current simulation waveform of the ZVS-DAB converter circuit model under SPS control condition, in which $U_{1}, U_{2}$ are the two-level square waves with a duty cycle of $50 \%$. Figure 7 is the simulation results of the ZVS-DAB traditional simplified circuit, only considering leakage inductance and phase-shift inductance. It can be seen that there is obvious waveform distortion in the start-up stage of the primary and secondary currents, and it takes longer to reach a steady state than the primary and secondary voltages. The common solution is to add start-up control to the hardware, which results in the complexity of the control algorithm, the reduction of power density, and the increase of system cost. In this paper, formula (6) is derived from the high frequency simplified circuit of DAB without start-up control, so that the primary and secondary currents can quickly attain a steady state.

Figure 8 shows the simulation waveform considering HF characteristics of the ZVS-DAB converter, namely, the parasitic capacitance of HFPT and winding loss. It can be seen that the waveform of the primary and secondary currents are obviously improved. The above simulation results indicate that the $\mathrm{HF}$ simplified mathematical model of the ZVS-DAB converter has better static stability than the traditional one.

4.2. Experimental Results of the ZVS-DAB Converter. In order to verify that the dynamic performance of the improved HF mathematical model of the ZVS-DAB converter is better than that of the traditional one, a $4.4 \mathrm{kw}, 40 \mathrm{khz}$ ZVS-DAB converter prototype based on $\mathrm{SiC}$ MOSFET has been constructed, and the experimental
TABLE 2: Model parameters of the simulation and experimental model.

\begin{tabular}{lc}
\hline Parameter & Value \\
\hline$V_{1} / V$ & 33 \\
$V_{2} / V$ & 9 \\
$C_{P} / \mu \mathrm{F}$ & 47 \\
$C_{S} / \mu \mathrm{F}$ & 110 \\
$R_{P} / \Omega$ & 0.2 \\
$L_{P} / \mu \mathrm{H}$ & 4.7 \\
$L_{S} / \mu \mathrm{H}$ & 2.2 \\
$\mathrm{~F} / \mathrm{KHz}$ & 40 \\
$R_{S} / \Omega$ & 0.05 \\
$K$ & 3 \\
\hline
\end{tabular}

prototype is demonstrated in Figure 9. In which HFPT, transformation ratio is $3: 1$, with good thermal performance and small size, is adopted. During the experiment, the primary side of the converter is powered by adjustable DC power supply, and the primary voltage is adjusted to $33 \mathrm{~V}$ (rated voltage is $30 \mathrm{~V}$ ); the secondary side is connected with adjustable resistance load, and the secondary voltage is adjusted to $9 \mathrm{~V}$ (rated voltage is $10 \mathrm{~V}$ ). DSP (TMS320F28335) and CPLD (Altera Max II) are utilized as a controller.

Figures 10 and 11 are the results of the tests on the prototype, which is shown in Figure 9. And we can get four groups of data by measuring currents and voltages of port $A B$ and port $C D$ in the HFPT box, and then the waveforms of Figure 10 are obtained via MATLAB software using these data sets. Similarly, through measuring currents and voltages of port ab and port cd in the HFPT box, we can get another four groups of data, and then the waveforms of Figure 11 are obtained via MATLAB software using these data sets. Figure 10 is the experimental waveform corresponding to the traditional mathematical model only considering leakage inductance and phase-shift inductance of the ZVS-DAB converter, in which the primary and secondary voltages oscillate violently at each switching point, and this caused waveform distortion. One of the reasons is that the DC bias makes the input and output current/voltage of the ZVS-DAB converter have large ripples; another reason is that the parasitic inductance of WBG semiconductors resonates with the parasitic capacitance of transformer and PCB, which causes obvious voltage spike [14]. Therefore, this paper focuses on the HF characteristics of the ZVS-DAB converter and deduces its HF mathematical model through adding resistance and capacitance to the circuit to suppress DC bias, thus reducing HF oscillation and waveform distortion of the circuit, stabilizing the input and output voltage waveforms. The experimental results are shown in Figure 11, we should take measures on the second reason, in order to eliminate the oscillation of the output waveform of the ZVS-DAB converter completely. 


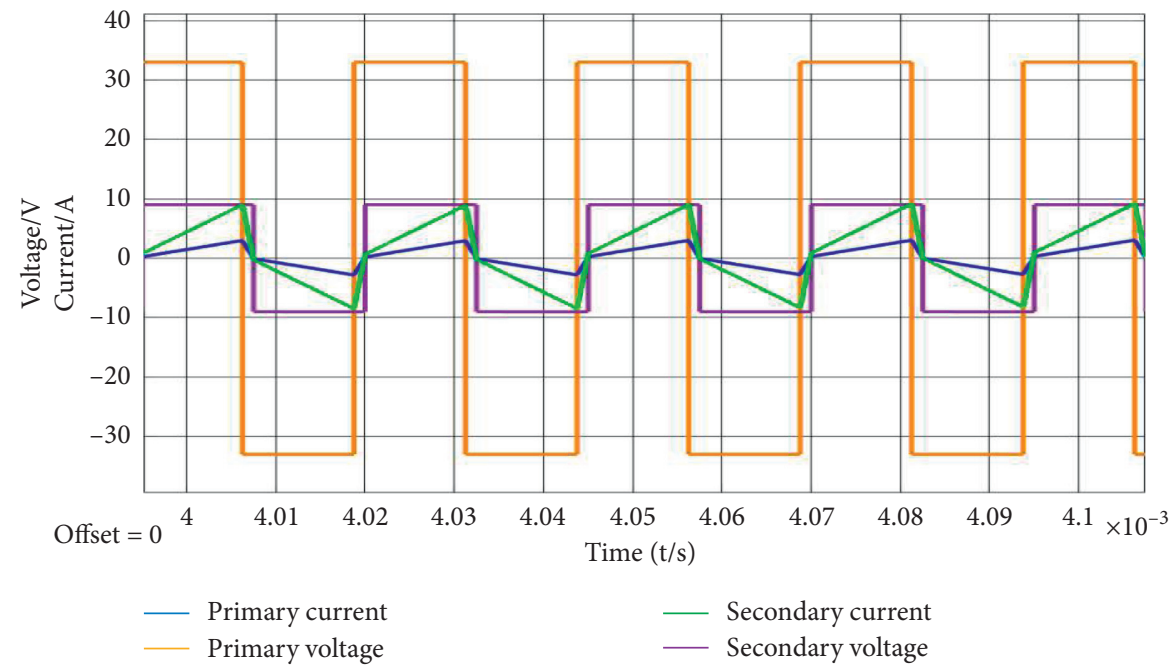

Figure 6: The simulation waveform of the ZVS-DAB traditional converter.

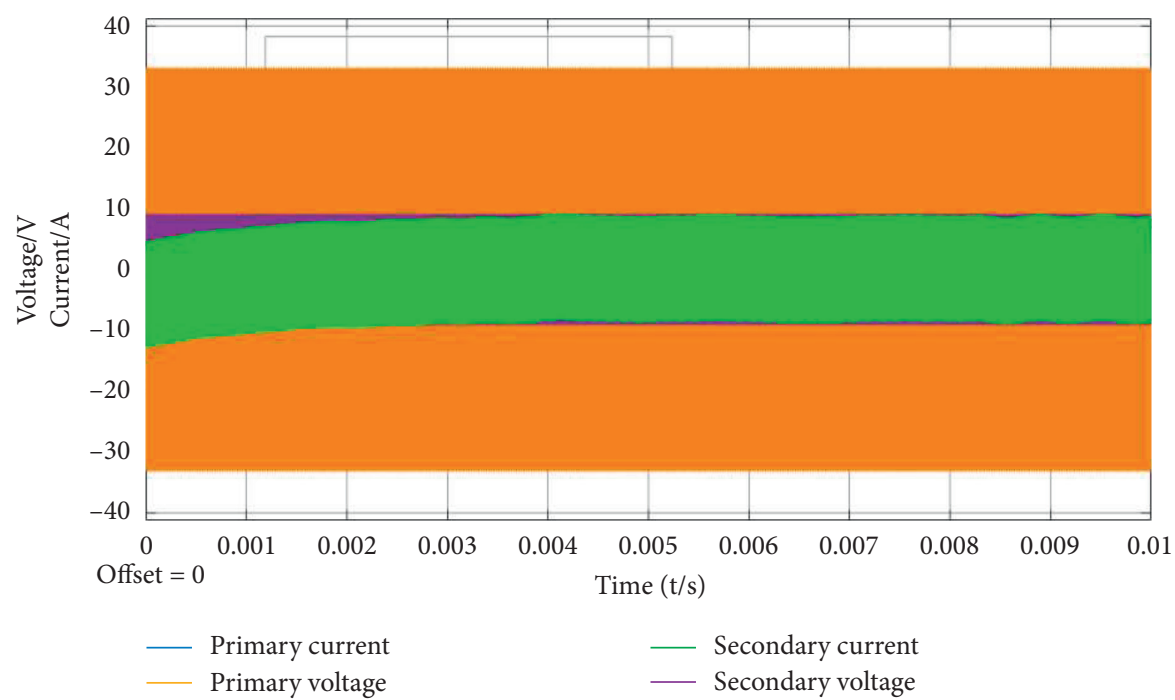

FiguRE 7: The system simulation waveform of the traditional ZVS-DAB converter.

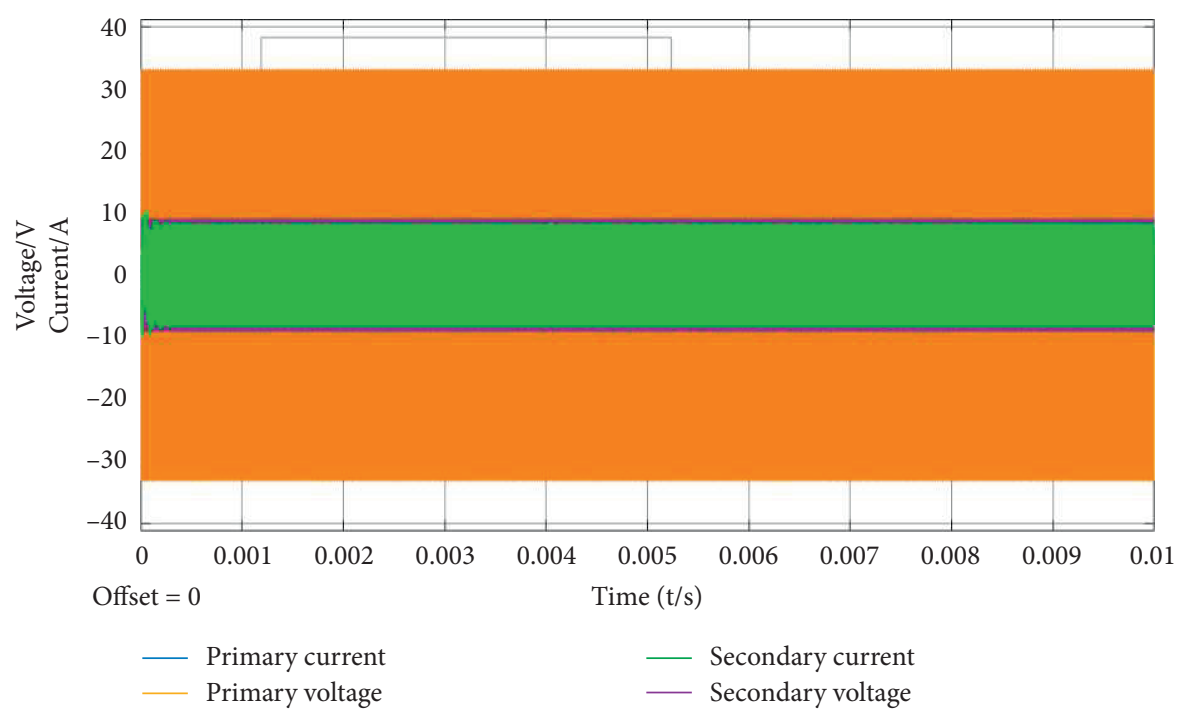

FIGURE 8: The system simulation waveform of the improved HF ZVS-DAB converter. 


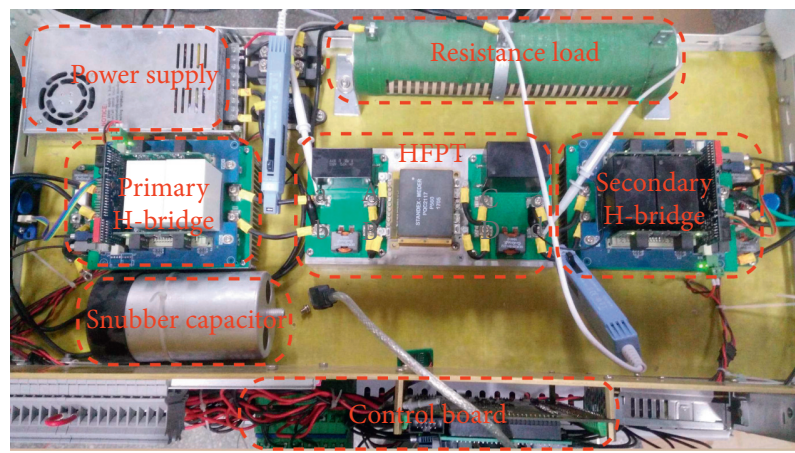

FIgURE 9: Prototype of the ZVS-DAB converter.

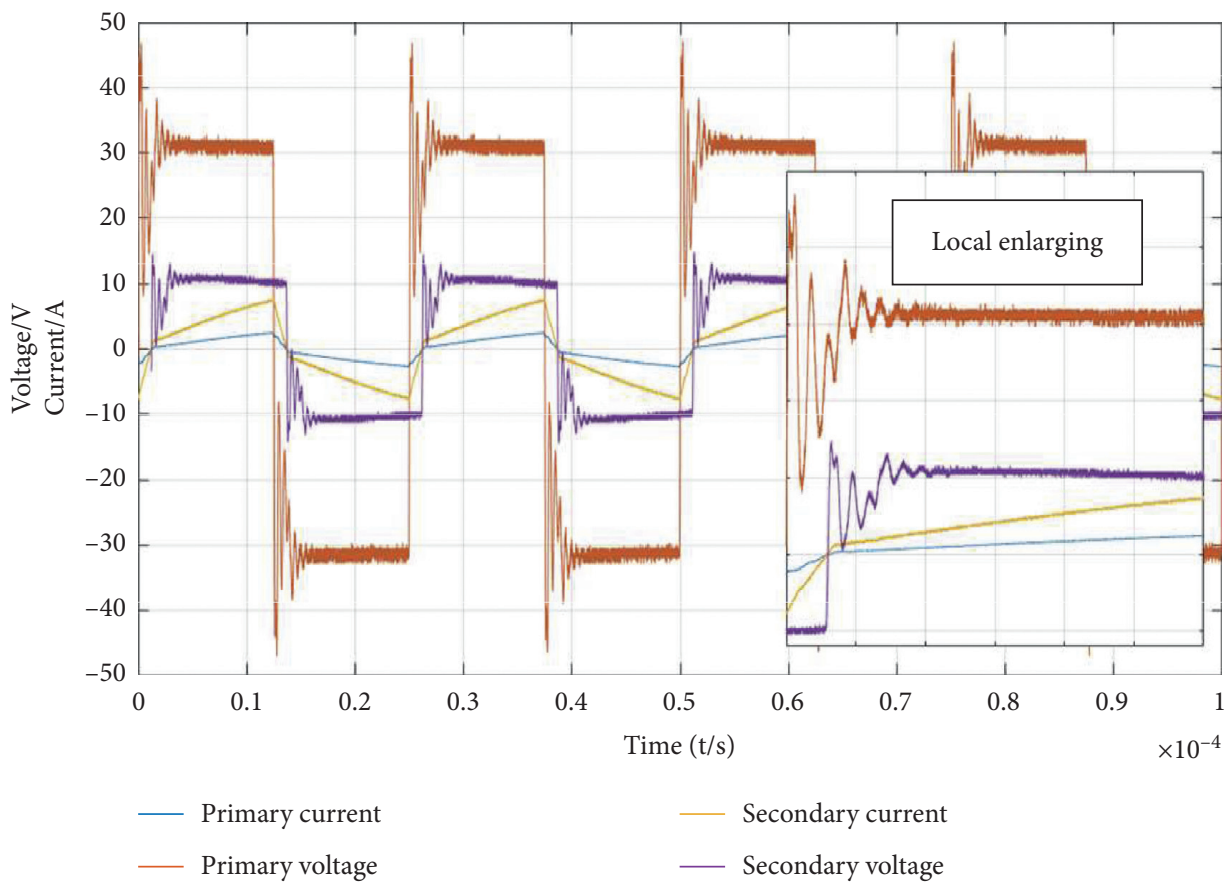

FIGURE 10: Experimental waveform of the traditional ZVS-DAB converter.

For example, first, we establish the simulation model of the ZVS-DAB converter on the basis of the oscillation wave in the experiment and deduce its mathematical expression, then find out the solution. It can be seen from the two local enlarged drawings in Figures 10 and 11 that the distortion, fluctuation, and oscillation of the original and secondary voltage waveforms of the proposed HF mathematical model of ZVS-DAB converter are much less than that of the traditional one, which further verifies the effectiveness of the proposed HF model of ZVS-DAB converter.

The results of the experimental data analysis, as shown in Figure 12, show that the DC bias of the primary voltage in the traditional ZVS-DAB model is the highest, and the total DC bias can be reduced by the improved ZVS-DAB converter model, which further proves that the proposed HF mathematical model of ZVS-DAB converter can improve the dynamic characteristics of the system. 


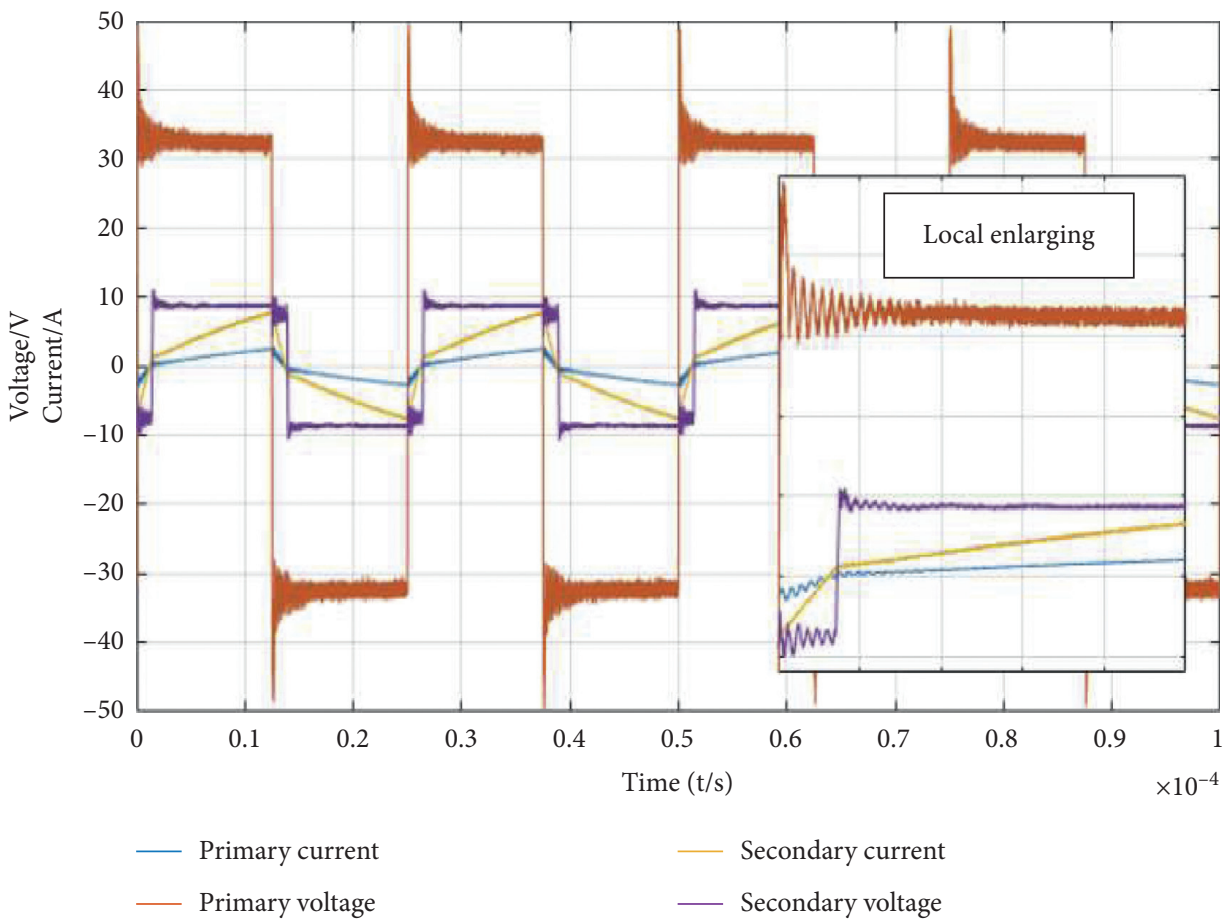

FIgURE 11: Experimental waveform of the improved HF ZVS-DAB converter.

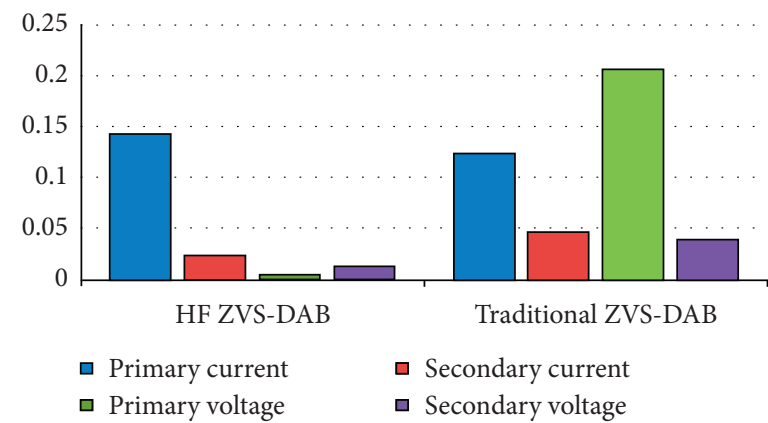

FIGURE 12: Experimental waveform of the improved HF ZVS-DAB converter.

\section{Conclusions}

This paper has fully considered the HF characteristics of the ZVS-DAB converter. And we have presented mostly used traditional simplified circuits for full bridge ZVS$\mathrm{DAB}$ converter and deduce their mathematical model, respectively, in this paper. On this basis, we propose a HF mathematical model, which take conduction loss and HF characteristics of the ZVS-DAB converter model into account. The stability of the HF circuit and traditional circuit of the ZVS-DAB converter is compared in simulation and experiment. Since the simulation model cannot fully reflect the real model, and the switches as well as the transformer utilized in the simulation cannot fully reflect HF oscillation caused by the parasitic parameters which arise during the HF scenario. In the end the steady-state performance of the ZVS-DAB converter is analyzed in the simulation, and the dynamic performance is studied in the experiment. It can be seen from the results of the simulation and experiment that the proposed HF mathematical model of ZVS-DAB converter, compared with the traditional one, can effectively reduce the DC component in the circuit and avoid the DC bias of the transformer which leads to magnetic saturation. Moreover, it can decrease the output waveform distortion rate, improve the system stability, reduce the output waveform oscillation rate of the primary and secondary side voltage, and advance the dynamic performance of the electric traffic energy system.

After analyzing the results from simulation and experiment, we can draw the following conclusions:

(1) The PSI of the ZVS-DAB converter should be on both sides of the transformer so that the PSI can play the role of filtering and restrain the HF oscillation of ZVS-DAB primary and secondary currents. 
(2) The HF oscillation of the ZVS-DAB converter can be suppressed through adding absorption capacitance to the WBG switches, but there will be current overcharge issues, or inserting parallel resistance at $\mathrm{H}$-bridge port to restrain $\mathrm{dv} / \mathrm{dt}$ by resistance energy consumption, or build a simulation model of the ZVS-DAB converter based on the oscillation wave in the experiment and deduce its mathematical expression to improve circuit topology or control algorithm, so as to reduce or eliminate the primary and secondary voltage oscillation of the ZVS-DAB converter.

\section{Data Availability}

The data used to support the findings of this study are available from the corresponding author upon request.

\section{Conflicts of Interest}

All the authors declare that there are no conflicts of interest regarding the publication of this paper.

\section{Acknowledgments}

The work was supported by the National Natural Science Foundation of China (Grant no. 51667020 and no. 61364010), the Outstanding Doctor Graduate Student Innovation Project of Xinjiang University (No. XJUBSCX2016018), and the Graduate Student Innovation Project of Xinjiang Uygur Autonomous Region (No. XJGRI2017025).

\section{References}

[1] Z.-B. Ya, Life Cycle Assessment of Energy Use, Carbon Emissions and Cost Benefit of Electric Vehicle, Tsinghua University, Beijing, China, 2016.

[2] M.-L. Fu, W.-T. Fei, and D.-K. Qi, "Research on wide voltage output vehicle power supply based on LLC topology," Mechanical and Electrical Engineering, vol. 8, pp. 911-915, 2017.

[3] L. Jing, Y.-L. Qiang, and G. Qing, "An efficiency optimization method of dual active bridge DC-DC converter based on loss model," Journal of Electrical Technology, vol. 32, no. 14, pp. 66-76, 2017.

[4] Y.-J. xi, L.-J. Qiang, and Z.-J. Fu, "Voltage balance control of power electronic traction transformer based on double active bridge DC-DC converter," Journal of Electrical Technology, vol. 31, no. 1, pp. 119-127, 2016.

[5] Z. Mariusz, K. Konstantin, R. Jacek, and B. Roman, "Design and evaluation of reduced self-capacitance inductor in DC/ DC converters with fast-switching $\mathrm{SiC}$ transistors," IEEE Transactions on Power Electronics, vol. 29, no. 5, pp. 24922499, 2014.

[6] X. Fei, Y.-R. Yang, and H.-Q. Alex, “A 98.3\% efficient GaN isolated bidirectional DC-DC converter for DC microgrid energy storage system applications," IEEE Transactions on Industrial Electronics, vol. 64, no. 11, pp. 9094-9103, 2017.

[7] R. W. A. A. De Doncker, D. M. Divan, M. H. Kheraluwala, and M. H. Kheraluwala, "A three-phase soft-switched highpower-density DC/DC converter for high-power applications," IEEE Transactions on Industry Applications, vol. 27, no. 1, pp. 63-73, 1991.
[8] C. Hong, Development of $15 \mathrm{kw}$ Phase Shifted Full Bridge ZVS Charger, Southwest Jiaotong University, Chengdu, China, 2012.

[9] S. Ling, L.-W. Jun, L.-Z. Qiang, and H. Jun, "Bilinear discretetime modeling and stability analysis of the digitally controlled dual active bridge converter," IEEE Transactions on Power Electronics, vol. 32, no. 11, pp. 8787-8799, 2017.

[10] Q. Hengsi and K.-W. Jonathan, "Generalized average modeling of dual active bridge DC-DC converter," IEEE Transactions on Power Electronics, vol. 27, no. 4, pp. 2078-2084, 2012.

[11] W.-S. En, Z.-Z. Dong, L. Chi, and W. Kui, "Time domain analysis of reactive components and optimal modulation for isolated dual active bridge DC/DC converters," IEEE Transactions on Power Electronics, vol. 34, no. 8, pp. 7143-7146, 2019.

[12] S. Kai, L.-Y. Jing, and W.-H. Fei, "Variable temperature parameter modeling of silicon carbide MOSFET," Chinese Journal of Electrical Engineering, vol. 33, no. 3, pp. 33-43, 2013.

[13] G.-Y. Shi, W.-Y. Jie, X.-D. Guo, and W. Wei, "A 1 MHz halfbridge resonant DC/DC converter based on GaN FETs and planar magnetics," IEEE Transactions on Power Electronics, vol. 32, no. 4, pp. 2876-2891, 2017.

[14] L. Bin, L. Qiang, and L.-C. Fred, "High-frequency PCB winding transformer with integrated inductors for a bi-directional resonant converter," IEEE Transactions on Power Electronics, vol. 34, no. 7, pp. 6123-6135, 2019.

[15] A.-S. Mohammad, S. Navid, and O. Martin, "LLC converters with planar transformers: issues and mitigation," IEEE Transactions on Power Electronics, vol. 32, no. 6, pp. 45244542, 2017.

[16] C. Bin, X. Peng, and J.-X. Hua, "Elimination of high frequency oscillation in dual active bridge converters by $\mathrm{dv} / \mathrm{dt}$ optimization," IEEE Access, vol. 7, pp. 55554-55564, 2019.

[17] Z. Biao, S. Qiang, L.-W. Hua, and Z.-Y. Ming, “Transient DC bias and current impact effects of high-frequency-isolated bidirectional DC-DC converter in practice," IEEE Transactions on Power Electronics, vol. 31, no. 4, pp. 3203-3216, 2016.

[18] G.-C. Yang, Research on Topology and Control of Power Electronic Transformer for Electric Traction, Tsinghua University, Beijing, China, 2015.

[19] S.-S. Li and L.-Z. Qiang, "Modeling and control method of three-phase double active bridge DC converter," Journal of Electrical Technology, pp. 1-13, 2019.

[20] G.-Z. Qiang, "Modulation scheme of dual active bridge converter for seamless transitions in multi working modes compromising ZVS and conduction loss," IEEE Transactions on Industrial Electronics, vol. 67, no. 9, pp. 7399-7409, 2019. 